\title{
Impact of Technology Spreading on MEMS design Robustness
}

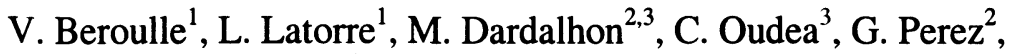 \\ F. Pressecq ${ }^{2}$, P. Nouet ${ }^{1}$ \\ 1 Laboratoire d'Informatique, de Robotique et de Microélectronique de Montpellier, France \\ 2 Centre National d'Études Spatiales, Toulouse, France \\ 3 European Aeronautic Defense and Space Company, Launch Vehicle Dpt., Paris, Franc
}

\begin{abstract}
This paper addresses the characterization of MEMS structures, industrially manufactured using front-side bulk micro-machining post-process techniques on CMOS dies. The systematic characterization of mechanical parameters, such as stiffness or mass, on a set of 100 cantilever devices provides us with ground knowledge concerning process parameter variations. Taking into account the foundry-given process parameter spreading such as the layer thickness uncertainties characterization results are compared with simulation results obtained using Monte-Carlo analysis in standard CAD environment. This study can be considered as a first step in the development of a global monolithic MEMS design methodology.
\end{abstract}

Key words: MEMS, Characterization, Process Scatterings

\section{INTRODUCTION}

An increasing interest is given to microsystems that monolithically integrate mechanical sensor parts with dedicated signal processing circuits $[1,2]$. The fabrication process usually relies on an industrial CMOS foundry followed by post-process releasing treatments for the fabrication of the mechanical elements. As a consequence, moving parts are made of CMOS materials such as silicon, oxides and aluminum. Since CMOS foundries neither characterize nor monitor mechanical properties of materials such as density or Young's modulus, particular design approaches have to be considered. 
The goal of this study is to characterize the scattering of mechanical parameters for basic cantilever structures made by an industrial FSBM (Front Side Bulk Micromachining) post-process on CMOS dies. The paper is organized as follows: the first part introduces the test chip used for the experimental study. The characterization protocol is presented in the second part. The third part summarizes and comments experimental results. Part four addresses design issues. AHDL modeling of mechanical structures is first stated. Then Monte-Carlo analysis is performed on mechanical devices using the CMOS layer thickness distribution given by the foundry. The soobtained statistical data is finally compared with experimental data.

\section{THE TEST CHIP}

In order to get experimental access to specific parameters, test samples have been designed and fabricated. The following sections introduce the so called U-Shape cantilever test device. Fabrication approach, and basic actuation principles (static and dynamic) are therefore developed.

The Front Side Bulk Micromachining (FSBM) post-process allows the fabrication of micrometric mechanical structures using a silicon wafer issued from a standard CMOS industrial process. This MEMS technology can be easily addressed in Europe through Multi-Project Wafer services of CMP [3]. CMOS is currently a $0.8 \mu \mathrm{m}$ or $0.6 \mu \mathrm{m}$ standard process with two or three metal layers from Austria Mikro Systems [4] while post-process is performed by IBS [5]. Since we have been using this fabrication facility for over 6 years we were able to observe a high process repeatability level.

Figure 1 represents a cross-sectional schematic of a CMOS process where polysilicon, metal and oxide layers are deposited on a substrate. According to designer requirements several etching masks can be superimposed to leave the silicon bulk uncovered at the end of the standard CMOS process. Then, the post-process operates as an anisotropic silicon etching that uses the various oxide layers as self-aligned masks. $<100>$ substrate planes can then be etched leaving the $<111\rangle$ planes of silicon substrate unaltered. In that way, suspended structures can be obtained as a heterogeneous stacking of various materials (namely Silicon Oxides, Polysilicon, Aluminum, Silicon nitrides). A set of 25 chips has been fabricated using AMS $0.8 \mu \mathrm{m}$ CMOS technology. As shown on figure 2, each chip includes 4 U-Shaped cantilever devices (namely C,D,E1,E2) and the corresponding readout circuitry. Figure 2 shows an example of such a structure after fabrication. Some important structure dimensions are reported in table 1 for further reference. 


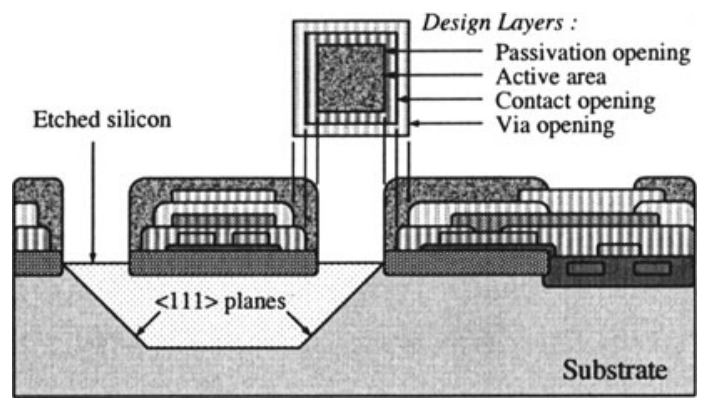

Figure 1. CMOS manufacturing process.

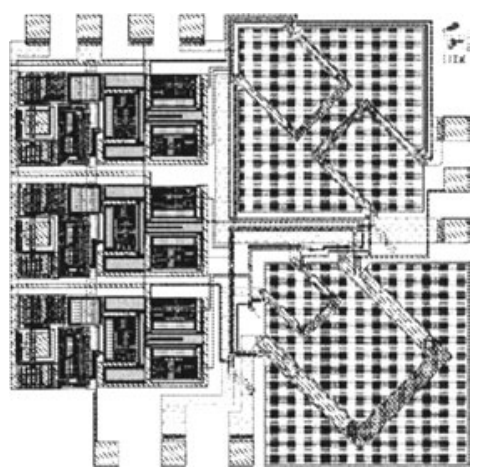

Figure 2. Layout of the whole test chip.

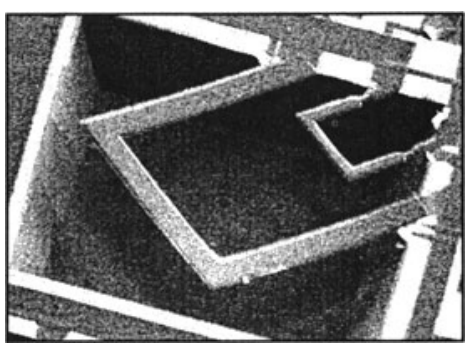

Figure 3. Example of U-Shape cantilever devices used for characterization

\begin{tabular}{|cc|c|c|}
\hline Device & LC & Wc & Wb \\
\hline C & 520 & 520 & 80 \\
\hline$D$ & 210 & 200 & 40 \\
\hline$E_{1} \& E_{2}$ & 320 & 320 & 40 \\
\hline
\end{tabular}

Table 1. Test structure dimensions in micrometers.

Our characterization technique is based on the U-shape cantilever beam that offers different actuation modes. Using a CMOS aluminum metal path in association with an external magnetic field, the device can be actuated by 
means of the Lorentz force (figure 4). In that case, the calibrated force can be static, using a constant electrical current, or dynamic.

In order to apply a known vertical displacement, a test probe driven by a micromanipulator is used. For each actuation mode, the device bending is electrically monitored via embedded polysilicon strain gauges and on-chip signal conditioning.

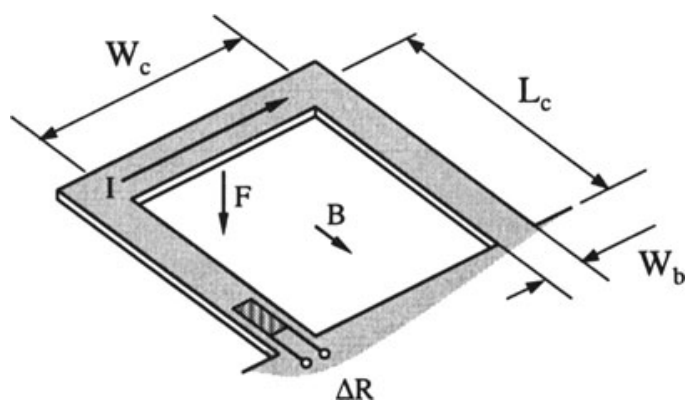

Figure 4. Magnetic actuation principle and reference dimensions.

\section{CHARACTERIZATION PROTOCOL}

\subsection{Static mode characterization}

The purpose of static characterization is to measure the cantilever stiffness. Since the stiffness relates to the force and the displacement, the cantilever is deflected (i) by applying a known displacement by means of a micromanipulator (figure 5) and (ii) by applying a known Lorentz force (figure 6).

While using the micromanipulator, it is very difficult to precisely determine when the contact between the probe and the structure occurs. In the extraction process, only the slope of the characteristic is considered so that this offset has no effect on the result.

In both cases, a linear response of the structure is observed as expected. From the measured slopes $A$ and $S$, we can calculate the stiffness $k$ as follow:

$$
k=\frac{F}{z}=\frac{A}{S}
$$




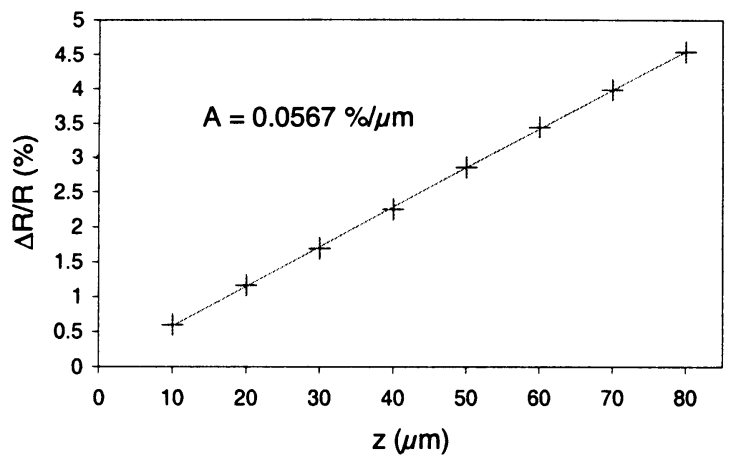

Figure 5. Static response versus displacement

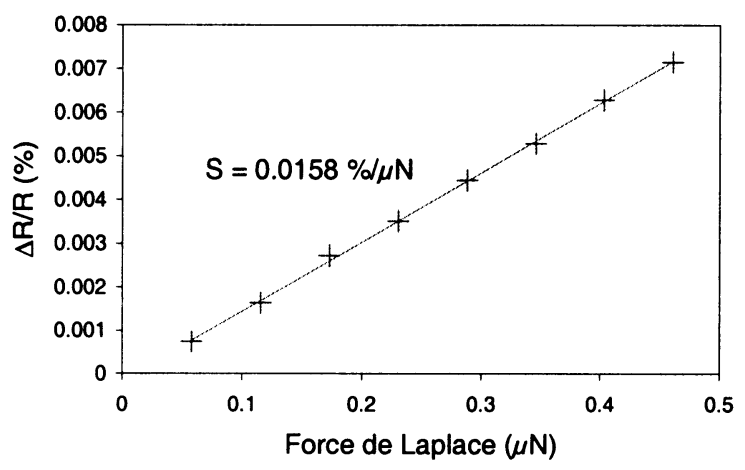

Figure 6. Static response versus force

\subsection{Dynamic mode characterization}

Some important dynamic parameters are the cantilever mass $\mathbf{M}$ and the damping factor $\mathrm{D}$. Those parameters can be calculated from the resonant frequency and the quality factor that are easily extracted on the frequency response. The frequency response is measured using a sinusoidal force current in association with a dc calibrated magnetic field. Experimentally, on-chip signal conditioning and an oscilloscope are used to get the output signal amplitude. However, results (figure 7) are presented here in term of peak-to-peak relative resistance variation to be consistent with previous results. 


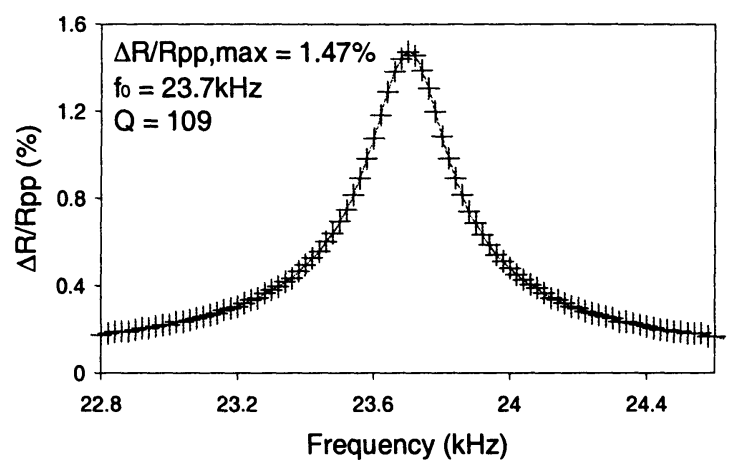

Figure 7. Frequency response

The mass can be calculated from the previously determined stiffness using the observed resonant frequency:

$$
M=\frac{k}{\left(2 \pi f_{0}\right)^{2}}
$$

The quality factor is then calculated using the definition of the $-3 \mathrm{~dB}$ bandwidth:

$$
Q=\frac{f_{0}}{\Delta f_{-3 d B}}
$$

Finally, the damping factor is calculated from both $k, M, f_{0}$ and $Q$ as follows:

$$
D=\frac{k}{2 \pi f_{0} Q}
$$

\subsection{Mechanical parameter validation}

Comparing actual digitized step response of the U-Shape cantilever with the calculated overshoot envelope validates both static and dynamic parameters. For low damped second order mechanical systems, the nth order overshoot amplitude $O_{n}$ is deduced from the following relation: 


$$
\ln \frac{O_{1}}{O_{n}} \approx(n-1) \frac{D}{2 M f_{0}}
$$

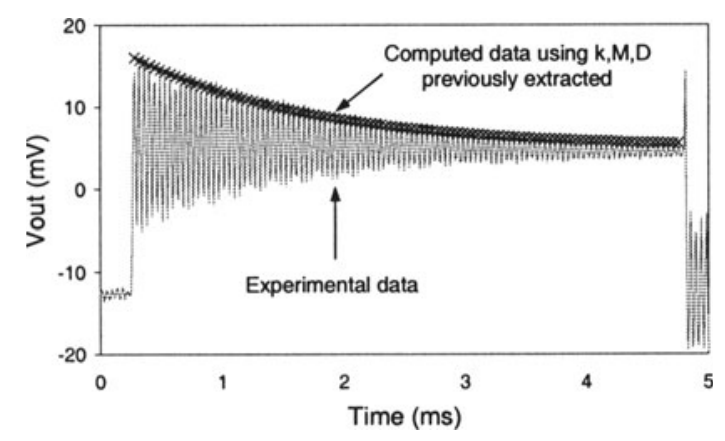

Figure 8. Comparison between experimental step response and calculated overshoot envelope.

Taking apart the first measurement involving human control of the test probe, the whole characterization protocol has been implemented onto an automatic test bench in order to reduce experimental condition spreading. The test bench is composed of standard test equipment (oscilloscope, supplies, function generator) controlled by a workstation using a standard IEEE488 instrumentation interface bus.

\section{CHARACTERIZATION RESULTS}

Table 2 summarizes the average value and standard deviation found for each of the extracted parameters. Since beams are made of a CMOS layer stack, reported deviations concerning Mass and Stiffness can be correlated with a known foundry uncertainty of about $20 \%$ given on each CMOS layer thickness.

For the beam stiffness, uncertainties of about 10 percents have been observed on the same fabrication run. One should keep in mind that from one run to another, standard deviations should be greater. Also, it is worth noting that standard deviation becomes generally smaller when the mechanical device size increases (the lowest deviations are obtained on the $\mathrm{C}$ cantilever). This result is not surprising given that it is a rule of thumb in the analog IC design field to increase dimensions in order to minimize process parameter distribution. 


\begin{tabular}{|l|c|c|c|c|c|c|}
\hline & \multicolumn{2}{|c|}{ C } & \multicolumn{2}{c|}{ E } & \multicolumn{2}{c|}{ D } \\
\hline & Avg. & $\begin{array}{l}\text { Std } \\
(\%)\end{array}$ & Avg. & $\begin{array}{l}\text { Std } \\
(\%)\end{array}$ & Avg. & $\begin{array}{c}\text { Std } \\
(\%)\end{array}$ \\
\hline$A(\% / \mu \mathrm{m})$ & 0.025 & 3.1 & 0.059 & 4.5 & 0.161 & 7.5 \\
\hline$S(\% / \mu \mathrm{N})$ & 0.011 & 7.2 & 0.015 & 8.6 & 0.017 & 14 \\
\hline$K\left(\mathrm{~N} \cdot \mathrm{m}^{-1}\right)$ & 2.4 & 10.3 & 3.9 & 13.1 & 9.5 & 14.1 \\
\hline$f_{0}(\mathrm{kHz})$ & 8.97 & 2.6 & 23.06 & 6.7 & 37.84 & 7.3 \\
\hline$Q$ & 59.07 & 4.7 & 110.02 & 7.0 & 159.12 & 19.7 \\
\hline$M\left(10^{-10} \mathrm{~kg}\right)$ & 7.53 & 6.9 & 1.86 & 4.8 & 1.67 & 7.7 \\
\hline$D\left(10^{-7} \mathrm{~N} \cdot \mathrm{m} \cdot \mathrm{s}^{-1}\right)$ & 7.19 & 7.4 & 2.46 & 10.1 & 2.4 & 10.8 \\
\hline$T_{G !}(\% / \mu \mathrm{N})$ & 0.0032 & 7.2 & 0.0038 & 8.6 & 0.0033 & 28 \\
\hline
\end{tabular}

Table 2. Characterization results

Robust design may only be achieved by taking into account parameter spreading. For illustration, an example of mechanical parameter scattering subsequent failure that has been observed with the device presented on figure 9. The matched cantilevers are designed in order to improve the device sensitivity to magnetic field by a factor two at resonant frequency. Cantilevers are then actuated with phase opposition and the four gauges are integrated into a single Wheatstone bridge for full sensitivity. In this particular non-robust design example, the expected improvement has never been reached on a set of 15 devices due to systematic mechanical parameter mismatch between the two structures. On figure 9, residual oxide on the corner of the lower U-Shape cantilever is undoubtedly the cause of a mass mismatch that produced the frequency response shown on figure 10.

\section{SIMULATIONS}

The way to improve design robustness is to take into account the process parameters variation at the design level. In the microelectronic design environment, this is achieved by performing Monte Carlo analysis, using the parameter distributions given by the foundry. Unfortunately, in the case of monolithic MEMS fabricated using industrial CMOS facility, no mechanical parameter distribution has been so far characterized. 


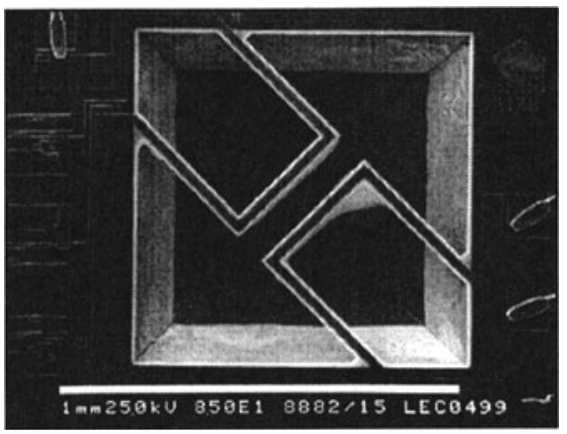

Figure 9: Residual oxides on matched cantilevers

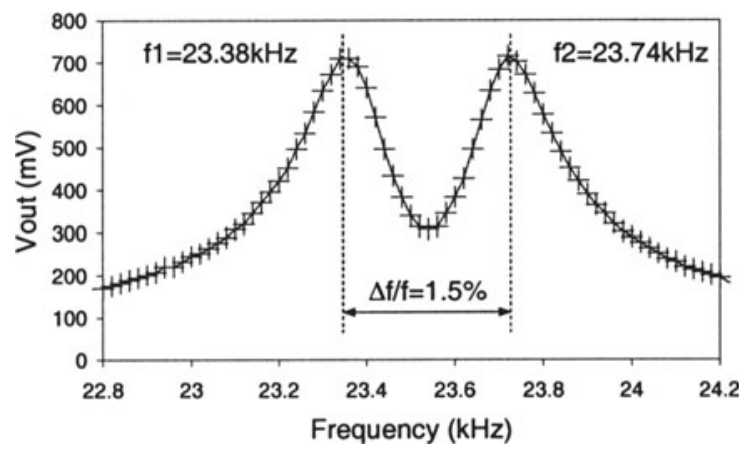

Figure 10. Frequency response on a pair of matched cantilevers : two different resonant frequencies are identified due to mass mismatch

The only information we were able to find in the foundry process parameter datasheet is the maximum, the average, and the minimum thickness for the various CMOS layers. Based on this information, a normal distribution for each layer thickness has been assumed and calibrated by taking one third of minimum (or maximum) thickness for the standard deviation. The so-obtained statistical data has been used in Monte Carlo analysis in association with an analog HDL model of the U-Shape device. It is worth noting that a special model has been developed for this purpose [6]. This model relates low-level parameters such as material properties and dimensions to the high-level behavior of the structure. An example of Monte Carlo analysis result is shown on figure 11 . The resonant frequency f0 has been computed 1000 times, taking into account the layer thickness distribution.

For C, E, and D cantilevers the same f0 standard deviation of $1.9 \%$ has been computed when deviations from $2.6 \%$ to $7.3 \%$ have been 
experimentally observed (table 2). This result is not surprising since simulation does neither consider material properties distribution (e.g. density or Young's modulus) nor post-process related uncertainties (e.g. remaining oxides).

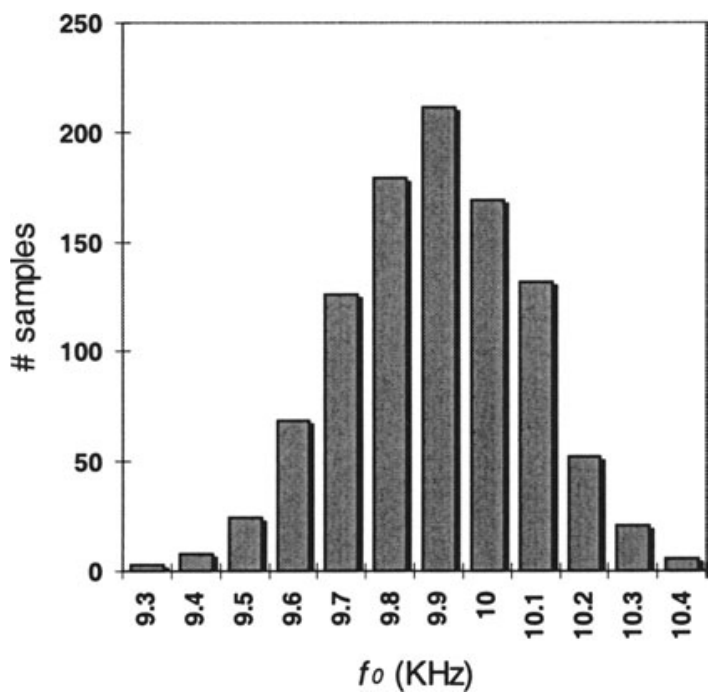

Figure 11: Monte Carlo analysis. The resonant frequency of the $\mathrm{C}$ cantilever has been computed 1000 times taking into account CMOS layer thickness distribution.

Realistic simulations would consider all the process parameter statistical distribution. This can be achieved by persuading the CMOS manufacturers to characterize and monitor several mechanical parameters. Obviously, such approach is not reasonable until CMOS microsystems rise significantly on the market. Another way is to use characterization data with some assumptions in order to get matched simulation results. Such approach will be developed as a further work.

\section{CONCLUSION}

In this paper, the robust design of MEMS monolithic devices based on CMOS process is addressed. In particular, a characterization methodology has been detailed in order to extract data that are not provided by traditional microelectronic manufacturer. This methodology involves a specific test structure, the U-Shape cantilever and does not require expensive equipments. Mechanical parameter such as stiffness, mass, damping factor or resonant 
frequency have been characterized on a set of about 400 structures, thus providing statistical evaluation of parameters spreading. The paper finally introduces the use of Monte Carlo analysis for MEMS design improvement. So far, only the layer thickness distribution has been considered in simulations. On going work concerns the use of experimental data to calibrate Monte Carlo analysis.

\section{REFERENCES}

[1]H. Xie, G.K. Fedder, “A CMOS-MEMS Lateral-axis Gyroscope”, proc. MEMS 2001, pp. 162,165 .

[2] “Single- and dual-axis micromachined accelerometers (ADXL150, ADXL250)", Analog Dialogue Journal, Vol. 30, n4, 1996.

[3] Circuits Multi-projets (CMP) is a broker in ICs, MCMs and MEMS. See http://cmp.imag.fr/

[4] See http://www.ams.co.at

[5] Ion Beam Services, ZI Peynier Rousset, Rue Gaston Imbert Prolongée, 13790 Peynier France - Email : ion-beam-services@wanadoo.fr

[6] L. Latorre, P. Nouet, Y. Bertrand, P. Hazard, F. Pressecq, "Characterization and modeling of a CMOS-compatible MEMS technology", Sensors and Actuators, Vol. 74, 1999, pp. 143-147. 